\title{
Research on Shanghai's Economic Influencing Factors Based on Grey Relational Analysis
}

\author{
Wang Lingyi ${ }^{1, *}$ \\ ${ }^{1}$ School of Data Science and Artificial Intelligence, Dongbei University of Finance and Economics, Dalian, Liaoning \\ Province, China \\ *Corresponding author. Email: duoerxs@163.com
}

\begin{abstract}
Affected by the new crown epidemic, the world economy has been in a long-term downturn, but the Chinese economy can achieve positive economic growth under this situation. As an important economic centre in China, studying the influencing factors behind Shanghai's economy is significant for improving China's economic development. This paper uses the grey relational analysis method to conduct an empirical study on the relationship between Shanghai's economic development, urban environment and other indicators. The study found a large degree of correlation between the various indicators in the index system and the economic development of Shanghai, but the correlation degrees corresponding to different indicators are different. Based on the influenced factors, this article puts forward policy recommendations for Shanghai's economic development, such as increasing environmental protection and provides a theoretical basis for promoting Shanghai's economic growth.
\end{abstract}

Keywords: Shanghai economy, index system, grey relational analysis, influencing factors.

\section{INTRODUCTION}

Since the new crown epidemic outbreak, the economies of various countries have been impacted to varying degrees, facing severe challenges. However, China's economy can achieve positive growth, closely related to China's efficient government policies. Economic growth is a catalyst for the country's overall development and studying the reasons for China's economic growth against the trend is of great significance to improving the quality of economic development. As an important economic centre of China, Shanghai's economic development can be regarded as a typical representative of China's economic development.

Shanghai enjoys the unique natural and geographical conditions. At the same time, Shanghai has a profound historical and cultural heritage, and the level of education, science, and technology is at the forefront of the world. As a magical capital with outstanding all-around development, Shanghai's economic development will inevitably receive national attention. Measures such as deepening reforms have further expanded Shanghai's economic scale and gradually developed into one of the country's top high-end cities. The rapid development of Shanghai has attracted many scholars to research Shanghai's economy. The research results analyse and study various factors affecting Shanghai's economy and propose corresponding policy measures.

\section{LITERATURE REVIEW}

In recent years, several researchers have continuously carried out various studies on factors affecting the country's economy. Zhang Jianguo et al. found that the coupling degree between household consumption and economic growth was very high through the grey relational analysis method and proposed correctly understanding the deep-seated effect of consumption on the relationship between economic growth and continuously improving consumption structure $^{[7]}$. Geng Liyan et al. used the grey relational analysis method to measure Hebei's logistics industry and regional economic development relevance and found that the secondary industry's added value has the most significant relevance to the logistics industry ${ }^{[8]}$. Lu Manyi constructed five evaluation indicators for development concepts, which used the grey correlation theory to measure the degree of correlation between technological innovation and the other four indicators ${ }^{[9]}$. It was made corresponding recommendations based on the empirical results. Jun He et al. studied the dynamic 
relationship between the direct financing structure and economic growth through the endogenous growth theory model and verified it by empirical research ${ }^{[9][10]}$. The direct financing structure has a nonlinear effect on China's economic development.

At the economic level of Shanghai, the following scholars have explored its influencing factors. By establishing a regression model, Ma Xiaoli found that the three major industries have a promoting effect on economic growth, and the promotion of the secondary and tertiary industries is much more important. Hao Yuanyuan et al. found that residents' consumption and minimum wage have a significant positive effect on Shanghai's economic growth through establishing a VAR model and other methods, and the effect of residents' consumption level is high ${ }^{[1][2]}$. Zhang Lili and others found that the amount of investment in real estate development and housing has a long-term role in promoting economic growth ${ }^{[3][4]}$. Xia Zufei et al. found a long-term equilibrium relationship between economic growth, power consumption, and upgrading and optimisation of the industrial structure through a modified error model ${ }^{[5]}$. From the perspective of sustainable development, they proposed that Shanghai's industrial structure reform should focus on modern service industries and continue to develop emerging industries. Kan Yudie et al. found that capital and labour are conducive to develop Shanghai's economy by establishing a Douglas production function model and that capital is significant to Shanghai's economy ${ }^{[6]}$.

In summary, scholars have done more research on economic influencing factors, and the research scope is broad. Their research results are reflected in both theoretical and empirical levels. Based on the existing literature's research on economic influencing factors, it can be seen that the existing literature has three shortcomings. First of all, the influencing factors of the research are relatively single. Most documents only select 1-3 influencing factors when studying the factors that affect the economy. However, the economic development of a region or a country is a complex process, and its influencing factors are diverse. It is too one-sided to select only a few factors for research. Secondly, some documents try to increase influencing factors to build an index system to study the relationship with the economy, but they fail to cover the whole range when selecting the index. After the indicators are classified, the number of different categories is relatively small, and it is difficult to form a comprehensive indicator system. Finally, based on the existing literature, it can be found that when scholars choose factors that affect the economy, they have conducted sufficiently specific studies on factors such as household consumption and the three major industries. There is a lack of research on factors such as urban environment, foreign trade, and health. Based on the research of the existing literature, this paper overcomes the above three shortcomings, selects influencing factors from multiple angles to construct an index system, and calculates the correlation between Shanghai's economy and each index in the index system through the grey correlation analysis method, and conducts it according to the results. In-depth analysis will provide a theoretical basis for the country to formulate corresponding policies, which will help further promote the prosperity and development of Shanghai's economy.

\section{DATA SELECTION}

This article selects Shanghai's GDP from 2009 to 2019 as an indicator reflecting Shanghai's economic development, environmental protection investment as an indicator of Shanghai's environmental conditions, total import and export volume as an indicator of foreign trade development, and scientific and technological achievements. As an indicator reflecting the level of science and technology in Shanghai, per capita disposable income and per capita consumption expenditure are selected as indicators to reflect the livelihood of Shanghai's people's livelihood, general public budget expenditures are selected as indicators to reflect financial expenditures, and total fixed assets in the whole society are selected as the indicators to reflect Shanghai. The city's investment indicators are selected from the original premium insurance income as an indicator reflecting the development of Shanghai's financial industry, the number of health institutions as an indicator reflecting the health situation in Shanghai, and the number of books published as an indicator reflecting the cultural development of Shanghai. It constructs an indicator system of Shanghai's economic influencing factors, as shown in Table 1. The data are from the "Shanghai Statistical Yearbook".

Table 1 Shanghai Index System

\begin{tabular}{ccc}
\hline Target layer & Element layer & Index layer \\
\hline \multirow{2}{*}{$\begin{array}{c}\text { Shanghai economy } \\
x_{0}: \text { Gross } \\
\text { product(100 million } \\
\text { yuan) }\end{array}$} & Environment & $x_{1}:$ Environmental protection investment (100 million \\
\cline { 2 - 3 } & Foreign trade & $x_{2}:$ Total import and export (100 million yuan) \\
\cline { 2 - 3 } & Technology & $x_{3}:$ Scientific and technological achievements (items) \\
\cline { 2 - 3 } & Finance & $x_{4}:$ General public budget expenditure (100 million yuan) \\
\hline
\end{tabular}




\begin{tabular}{cr}
\hline Investment & $\begin{array}{c}x_{5}: \text { Total investment in fixed assets of the whole society } \\
(100 \text { million yuan })\end{array}$ \\
\hline People's livelihood & $x_{6}:$ Per capita disposable income (yuan) \\
\cline { 2 - 2 } Financial industry & $x_{8}:$ Per capita consumption expenditure (yuan) \\
\hline Hygiene & $x_{9}:$ Number of health institutions (a) \\
\hline Culture & $x_{10}:$ Number of books published (species)
\end{tabular}

As shown in Figure 1, in addition to scientific and technological achievements, the values of other indicators have shown an increasing trend year by year from 2009 to 2019. Shanghai's GDP has steadily increased, indicating that Shanghai's economic scale has continued to expand. The amount of investment in environmental protection has increased yearly, which shows that Shanghai has continuously increased its emphasis on environmental protection. Shanghai's total import and export volume has shown an upward trend yearly, which indicates that Shanghai's foreign trade scale has been expanding and international economic exchanges have become more frequent. The number of scientific and technological achievements has shown a trend of first rising and then falling, indicating that the level of science and technology has fluctuated in recent years. General public budget expenditures have increased year by year, indicating that as the economy grows, the government's expenditures in all aspects have increased. The total investment in fixed assets of the whole society has steadily increased, indicating that the investment situation is good and the scale of investment by enterprises and governments has expanded. The rapid growth of per capita disposable income and per capita consumption expenditure indicates that residents' living standards have been continuously improved. The original insurance premium income has increased year by year, indicating that the financial industry is developing continuously. The number of health institutions has increased steadily, indicating that the urban health situation is continuously improved. The number of book publications has increased yearly, indicating that the urban cultural level is steadily improving.

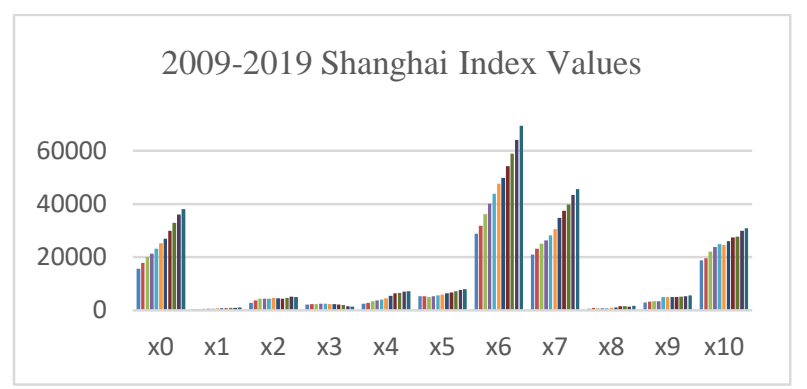

Figure 1 Shanghai Index System

\section{MODEL SELECTION}

This paper uses the grey correlation analysis method to study the correlation between Shanghai's economy and the index system composed of the urban environment, foreign trade, etc. Grey system theory is a marginal subject founded by Deng Julong in 1982. The grey system uses colour shades to reflect the amount of information. If a system is black, it means that the system is a black hole and has too little information. A system that is white means that the system is clear and has sufficient information. The system between black and white is the grey system, or the system with incomplete information referred to as the grey system. The grey correlation analysis method is based on the degree of similarity or difference between the development trends of factors, the "grey correlation degree", as a method to measure the degree of correlation between factors.

The steps of grey relational analysis are as follows:

(1) Determine the time series. Suppose the reference series is

$x_{0}=\left\{x_{0}(k) \mid k=1,2, \cdots n\right\}$

$k$ represents the time. Set $m$ comparison series, suppose the comparison series is

$x_{0}=\left\{x_{0}(k) \mid k=1,2, \cdots n\right\}, i=1,2 \cdots m$.

This article selects Shanghai's GDP as a reference series and takes each index value in the index system as a comparison series.

(2) Initialise the data. Due to the different physical meanings of various factors in the system, the data dimensions are not necessarily the same, which is not convenient for comparison, or it is challenging to get correct conclusions when comparing. Therefore, in the analysis of grey correlation degree, non-dimensional data processing should be carried out. Since each indicator of the system is a positive indicator, and it is passed

$x_{0}(t)=x_{0}(t) / x_{0}(1), x_{i}(t)=x_{i}(t) / x_{i}(1)$ 
to initialise the original sequence.

(3) Find the absolute difference series, the two-level minimum difference, and the two-level maximum difference between the comparison series and the reference series. The absolute difference sequence is

$$
\left|x_{0}(t)-x_{s}(t)\right|
$$

The minimum difference between the two levels is

$$
\min _{s} \min _{t}\left|x_{o}(t)-x_{\mathrm{s}}(t)\right|
$$

The maximum difference between the two levels is

$$
\max _{s} \max _{t}\left|x_{o}(t)-x_{\mathrm{s}}(t)\right| \cdot
$$

(4) Calculate the correlation coefficient.

$$
\xi_{i}(k)=\frac{\min _{s} \min _{t}\left|x_{0}(t)-x_{s}(t)\right|+\rho \max _{s} \max _{t}\left|x_{0}(t)-x_{s}(t)\right|}{\left|x_{0}(k)-x_{i}(k)\right|+\rho \max _{s} \max _{t}\left|x_{0}(t)-x_{s}(k)\right|}
$$

It is the resolution coefficient to compare the correlation coefficient of the sequence $x_{o}$ to the reference sequence $x_{i}$ at $k$ time. $\rho \in[0,1]$ is the resolution coefficient. Generally speaking, the larger the resolution coefficient is, the greater the resolution is; the smaller the resolution is, the smaller the resolution is. This paper takes $\rho$ as 0.5 .
(5) Calculate the degree of relevance. The degree of relevance is to concentrate the relevance coefficients at each moment into an average value.

$r_{i}=\frac{1}{n} \sum_{k=1}^{n} \xi_{i}(k)$

(6) Sort the degree of relevance. When there are $m$ comparison numbers, there are also $\mathrm{m}$ relative correlation values arranged according to their value, which is the correlation order. The degree of relevance directly reflects the pros and cons of each comparison sequence to the reference sequence.

\section{EMPIRICAL ANALYSIS}

This article selects Shanghai's 2009-2019 GDP as the dependent variable, selects 10 indicators of the same period as independent variables (explanatory variables), uses matlab2014 to calculate the grey correlation degree of the data and ranks the correlation degree according to the results, and analyses The relationship between Shanghai's economy and urban environment, foreign trade, technological level and other indicators. The greater the correlation degree is, the closer the relationship between the variables is. The calculation results are shown in Table 2.

Table 2 Calculation results of grey correlation degree

\begin{tabular}{ccc}
\hline Independent variable & Correlation & Sort \\
\hline Environmental protection investment & 0.9079 & 2 \\
Total import and export & 0.7996 & 6 \\
Technological Achievements & 0.6530 & 10 \\
General public budget expenditure & 0.7900 & 7 \\
Total investment in fixed assets of the & 0.6811 & 9 \\
whole society & & \multicolumn{2}{|}{} \\
Disposable income per capita & 0.9667 & 3 \\
Per capita consumption expenditure & 0.8866 & 4 \\
Original insurance premium income & 0.8508 & 4 \\
Number of health institutions & 0.8274 & 5 \\
Number of books published & 0.7635 & 8 \\
\hline
\end{tabular}

It can be seen from Table 2 that the correlation between these 10 indicators and Shanghai's economy exceeds $65 \%$, indicating that these 10 indicators have a relatively close relationship with Shanghai's economy. The strength of the correlation between each indicator and Shanghai's economy is: per capita disposable income $>$ environmental protection investment> per capita consumption expenditure $>$ original insurance premium income $>$ a number of health institutions $>$ total import and export $>$ general public budget expenditure $>$ a number of book publications $>$ fixed assets of the whole society Total investment> scientific and technological achievements.

Per capita disposable income and per capita consumption, as indicators that reflect the livelihood of Shanghai's people, are highly correlated with Shanghai's economy. Their relevance is 0.9667 and 0.8866 respectively, indicating that people's livelihood is closely related to Shanghai's economy. In recent years, most citizens are more satisfied with their current lives. However, with rising prices and higher employment requirements, some citizens feel unsatisfied with the current life situation. 
As an indicator reflecting the urban environment of Shanghai, the amount of environmental protection investment correlates with Shanghai's economy of 0.9079 , indicating that the urban environment has a very obvious effect on promoting economic growth. Although Shanghai's ecological environment has improved significantly in recent years, air and water pollution still needs further improvement. A high-quality urban environment can effectively promote the development of the urban economy. Therefore, it is necessary to increase the protection of the urban environment.

As an indicator reflecting the development of Shanghai's financial industry, the original insurance premium income has a high degree of correlation with the Shanghai economy, reaching $85 \%$, indicating that Shanghai's financial industry has an essential role in promoting the economy. Shanghai is an important financial centre of the country, and its financial industry is at the forefront of the world. The developed financial industry has played a significant role in driving the city's economy. In the future development process, we should continue to attach importance to the development of the financial industry.

The number of health institutions is used as an indicator to reflect the health situation in Shanghai, and its correlation with Shanghai's economy exceeds $80 \%$, indicating that the health situation is closely related to the economy. A sufficient number of health institutions ensure to a certain extent the basic requirements for citizens to seek medical treatment and help protect the health of citizens. Based on citizens having a healthy body, can all walks of life be ensured, and economic growth can be promoted.

The total value of imports and exports is an indicator that reflects Shanghai's foreign trade situation, and its correlation with Shanghai's economy is close to $80 \%$. It shows that the relationship between Shanghai's economy and foreign trade is relatively close. Shanghai is an important port city in the country, and its trade scale has been expanding year by year, which has become an essential factor driving economic growth. Given the importance of foreign trade to economic growth, Shanghai should continue to expand the scale of foreign trade in the future.

As an indicator reflecting Shanghai's fiscal expenditure, general public budget expenditure is closely related to Shanghai's economy by $80 \%$, and its role in promoting the economy is also very significant. Shanghai's fiscal expenditure involves many aspects, and the amount of expenditure is increasing year by year. Combined with other indicators, it can be seen that the Shanghai government can increase the proportion of expenditures on people's livelihood and environmental protection, and further promote the development of Shanghai's economy.
The number of books published as an indicator reflecting the cultural situation of Shanghai has a greater degree of relevance to the economy of Shanghai, and attention should be paid to the role of culture in promoting the economy. Culture can mould citizens' sentiments and improve citizens' overall quality, which is conducted to cultivate high-quality talents, play the role of talents, and promote economic development.

As an indicator reflecting the investment situation in Shanghai, the total investment in fixed assets of the whole society has a high degree of relevance to Shanghai's economy, indicating that the improvement of the investment situation is conducive to economic development. At the government level, investment in infrastructure should be increased to improve people's livelihood and ultimately promote economic growth. At the enterprise level, investment structure should be optimised, investment efficiency should be improved, and business performance should be improved.

The number of scientific and technological achievements is an indicator that reflects the level of science and technology in Shanghai. Although the correlation with Shanghai's economy is ranked last, it is still relatively large in value, indicating that the relationship between them is also relatively close. As the economy grows, more workforce and material resources are invested in scientific and technological development. However, the number of scientific and technological achievements in Shanghai has declined in recent years, reflecting the lack of emphasis on scientific and technological development in Shanghai.

\section{CONCLUSIONS AND RECOMMENDATIONS}

This paper selects 10 indicators to construct an indicator system, covering people's livelihood, environment and other aspects. According to the results of empirical analysis, it can be concluded that there is a relatively close relationship between the various indicators in the indicator system and Shanghai's economy. We can start with these factors that affect Shanghai's economy and formulate related government policies to further promote Shanghai's economic.

(1) Improve people's livelihood conditions and increase social welfare

People's livelihood is the foundation of economic development. We should improve the housing conditions of the masses, continue to implement low-rent housing and appropriately raise the subsidy standard for low-rent housing for families in need. Efforts should be made to increase employment opportunities, use entrepreneurship to drive employment, take employment expansion as the top priority of safeguarding to improve people's livelihood, optimise the entrepreneurial environment, and provide the people with a stable source of income and livelihood security. 
Comprehensively promote residents' social endowment insurance and medical insurance, and establish a complete social security system.

(2) Increase environmental protection and build a beautiful city

An excellent urban environment is the foundation of economic development. We should expand the proportion of environmental protection investment in fiscal expenditures, increase urban green areas, and carry out the in-depth transformation of key polluted areas. For companies that discharge pollutants in violation of regulations, penalties should be increased. The company's pollution discharge should be strictly controlled, and environmental law enforcement should be continuously strengthened. Vigorously promote domestic waste classification, increase environmental protection publicity, cultivate citizens' awareness of environmental protection, encourage citizens to exercise supervision rights, and promptly stop behaviours that pollute the environment.

(3) Improve the financial market and integrate high-tech

Finance is the lifeblood of economic development. Based on the highly developed financial industry, it is necessary to pay attention to adequate supervision of the financial market, to ensure the order of transactions in the financial market, and to increase penalties for violations of laws and regulations, such as market manipulation and high-interest lending. Encourage the integration of the financial industry with high-tech technologies such as big data and artificial intelligence, and use science and technology to promote the development of the financial industry and stimulate the potential vitality of the financial industry.

(4) Strengthen opening up and expand international trade

Developed foreign trade is a catalyst for economic development. With economic globalisation and regional integration, Shanghai's import and export scale has become more extensive, the scope of transactions has been broader, and the transaction frequency has increased. On this basis, we must further focus on improving the trading environment and building a legalised business environment. Speed up the development of service trade, take the initiative to adapt to the new normal of economic development, and build a new open economic system. Reduce taxes on import and export enterprises, reduce the economic pressure on such enterprises, increase subsidies, and encourage exports.

(5) Improve infrastructure and optimise investment structure

Financial expenditure is the lubricant of economic development. The fiscal revenue of Shanghai has been increasing year by year, especially in recent years. The fiscal expenditure should also increase accordingly. Meanwhile, attention should be paid to optimising fiscal expenditure structure, which increases investment in medical care, transportation, etc. It encourages medical care, transportation, big data, artificial intelligence, and other technologies. It improves urban infrastructure construction, strengthening financial capital management, and improves investment efficiency.

\section{REFERENCES}

[1] Ma Xiaoli. The relationship between industrial structure and economic growth: Taking Shanghai as an example $[\mathrm{J}]$. Foreign Economic Relations and Trade, 2021, $\{4\}(04):$ 75-79.

[2] Hao Yuanyuan, Cao Hongzhong. Research on the Impact of Minimum Wage and Resident Consumption on Shanghai's Economic Growth [J]. Commercial Economy, 2020, \{4\}(07): 27-30.

[3] Zhang Lili, Li Qianfei. The impact of real estate market fluctuations on important macroeconomic indicators: Taking Shanghai as an example [J]. Shanghai Real Estate, 2020, \{4\}(12): 16-22.

[4] Chen Liang, Tan Qian. The Impact of the Regional Comprehensive Economic Partnership Agreement (RCEP) on Shanghai's economy and countermeasures[J]. Scientific Development, 2021, $\{4\}(06): 41-47$.

[5] Xia Zufei, Wang Meng, He Wen. An Empirical Study on the Relationship between Shanghai's Industrial Structure, Power Consumption and Economic Growth[J]. China Business Review, 2021, \{4\}(03): 42-47.

[6] Kan Yudie, Luo Fang. The impact of capital and labor on Shanghai's economic growth[J]. Economic Research Guide, 2019(06): 130+135.

[7] Zhang Jianguo, Liu Qiuxiu, Xiang Renkang. An Empirical Study on the Impact of Resident Consumption on my country's Economic Growth [J]. Business Economics Research, 2021(14): 58-61.

[8] Geng Liyan, Hu Rui, Zhang Zhanfu. Research on the Relevance of Hebei Province's Logistics Industry and Regional Economic Development [J]. Chinese Market, 2021(16): 4-6.

[9] Lu Manyi. Analysing the economic development quality of the western region based on the grey relational theory[J]. Shangxun, 2021(05): 17-18.

[10] Yuan Ruhua, Zang Yanqiu. An empirical analysis of the coupling and coordination of economic development and water resources environment in 
the Yangtze River Economic Zone[J]. Water /10.16381/j.cnki.issn1003-207x.2020.0114. Conservancy Economics, 2021, 39(02): 1-8+95.

[11] He Jun, Han Wenyan, Bi Gongbing. The Nonlinear Impact of Direct Financing Structure on Economic Development[J/OL]. Chinese Management Science: 1-12[2021-07-26].

https://doi.org 